Rabaska

Revue d'ethnologie de l'Amérique française

\title{
La musique traditionnelle acadienne : au carrefour des cultures française et celtique
}

\section{Robert Richard}

Volume 12, 2014

URI : https://id.erudit.org/iderudit/1026801ar

DOI : https://doi.org/10.7202/1026801ar

Aller au sommaire du numéro

Éditeur(s)

Société québécoise d'ethnologie

ISSN

1703-7433 (imprimé)

1916-7350 (numérique)

Découvrir la revue

Citer ce document

Richard, R. (2014). La musique traditionnelle acadienne : au carrefour des cultures française et celtique. Rabaska, 12, 233-237.

https://doi.org/10.7202/1026801ar d'utilisation que vous pouvez consulter en ligne. 


\section{La musique traditionnelle acadienne : au carrefour des cultures française et celtique}

ROBERT RICHARD

Centre d'études acadiennes Anselme-Chiasson

Préalablement à la tenue du colloque, à l'automne 2010, l'ethnomusicologue Dariush Zarbafian, chargé de cours au Département de musique à l'Université de Moncton, avait organisé avec ses étudiants un séminaire-concert sur la musique traditionnelle acadienne, avec comme invité le violoneux acadien Eddie Poirier. Dans la même période, Dariush Zarbafian avait même lancé publiquement l'idée de la création d'un centre d'ethnomusicologie de la musique acadienne. En parallèle, l'ethnologue Ronald Labelle de la Chaire de recherche McCain en ethnologie acadienne avait discuté du sujet avec Barry-Jean Ancelet, de Louisiane. Une relation de travail avait également été créée avec le Centre d'études acadiennes Anselme-Chiasson.

Dans une suite logique des choses, pour rassembler les chercheurs et étudiants qui avaient un intérêt commun pour la chanson et la musique traditionnelle acadienne, Dariush Zarbafian et Ronald Labelle avaient pris l'initiative de créer un groupe de travail pour développer des activités de recherche et de diffusion de cette tradition. Lors d'une première réunion en janvier 2012, les participants avaient convenu d'un commun accord de prioriser la tenue du tout premier colloque sur la musique acadienne. Étalé sur deux volets, le premier théorique et l'autre pratique, la première partie s'est transportée naturellement vers l'Université du Cap-Breton (CBU), où Ronald Labelle venait d'obtenir un poste de professeur agrégé. C'est de là que, de concert avec des membres de la communauté universitaire, il avait poursuivi à distance l'organisation du premier colloque qui s'est déroulé du 11 au 13 octobre 2013.

Le doyen Stephen Augustine de l'Unama'ki College \& Aboriginal Learning, situé à $\mathrm{CBU}$, a d'abord souhaité la bienvenue aux 60 participantes et participants dans la région d'Unama 'ki (mot micmac : pays brumeux). Comme première séance du colloque et conférence d'ouverture, intitulées La musique et le patrimoine immatériel, le directeur Richard MacKinnon du Centre d'études du Cap-Breton s'est servi du Canada pour décrire les nombreuses initiatives tant locales que provinciales pour la sauvegarde 
du patrimoine culturel immatériel, même si le Canada n'avait pas encore reconnu officiellement les bienfaits et l'importance d'entériner la convention de l'Unesco en 2003. L'archiviste Catherine Arseneau et la directrice Jane Arnold de l'Institut Beaton (IB) ont brossé l'historique de projets ayant servi à l'acquisition de connaissances pour surmonter les défis de la migration analogique-numérique des documents audio et audiovisuels conservés à l'IB. Cela a eu comme conséquence la création du site Web Cape Breton's Diversity in Unity (Acadian, Mi'kmaq, Gaelic, Mining), qui illustre le rôle unificateur de la musique. L'archiviste en ethnologie acadienne Robert Richard du Centre d'études acadiennes Anselme-Chiasson a pour sa part donné quelques exemples d'acquisitions récentes de documents sonores, obtenus à la fois de façon active et passive ; ce qui apporte parfois son lot de surprises, tant du point de vue du contenu et que du traitement archivistique.

Dans la deuxième séance, qui avait pour titre La chanson et l'identité culturelle, le doctorant en études littéraires Louis-Martin Savard de 1'Université de Moncton a voulu mettre en relief le parcours intellectuel du folkloriste Joseph-Thomas LeBlanc et cela par l'intermédiaire des nombreuses communications et collaborations qu'il avait eues avec les ethnologues, les folkloristes, ses informateurs ainsi que les lecteurs de ses chroniques publiées entre 1938 et 1941 dans son journal, La Voix d'Évangéline. Ronald Labelle de $\mathrm{CBU}$ a présenté plusieurs exemples de récupération et de popularisation de chansons traditionnelles acadiennes et québécoises qui ont résisté à l'oubli ou qui sont restées populaires grâce à l'imprimé (ex. : recueils de chansons), aux technologies modernes (ex. : radios communautaires), ou encore à cause de l'apport des ethnologues et des folkloristes, des communautés, de chansonniers et des groupes de musique du renouveau. L'ethnomusicologue Jeanette Gallant de l'Université de Windsor a présenté l'étude qu'elle avait menée sur des chansons folkloriques acadiennes, dans trois contextes historiques marquants de l'histoire de l'Acadie, et l'importance qu'elles ont eues sur la construction identitaire nationale des Acadiennes et des Acadiens.

À l'intérieur de la troisième séance qui avait pour titre Aspects stylistiques des traditions musicales, les ethnomusicologues Beverley Diamond, Kati Szego et Meghan Forsyth de l'Université Memorial de Terre-Neuve ont peint un tableau des défis de produire un film documentaire, à la fois d'un point de vue technique et ethnographique. Elles ont présenté des extraits où l'accordéoniste terre-neuvien Stan Pickett retraçait l'histoire de l'accordéon et des danses carrées à Terre-Neuve. Pour sa part, Jean Duval du cégep de Victoriaville a mis en lumière les éléments asymétriques des reels détournés (crocheted tunes) dans la musique traditionnelle acadienne et québécoise ayant comme toile de fond ses recherches doctorales sur le même sujet. 
Lors de la quatrième séance, intitulée Perspectives ethnomusicologiques, l'ethnomusicologue Marlène Belly de l'Université de Poitiers s'est livrée à une présentation de la chanson-type Les Filles de La Rochelle, d'après des collectages effectués en France ainsi qu'au Canada français, et de l'importance de pouvoir accéder à distance aux ressources électroniques, ce qui facilite grandement une analyse comparative approfondie des similarités et des différences culturelles. Peter G. Toner de l'Université Saint-Thomas s'est permis de tracer le chemin parcouru dans la musique traditionnelle par les Écossais du Cap-Breton, de même que les Irlandais et les Acadiens du Nouveau-Brunswick; à son avis, les Irlandais n>ont pas su tirer profit des opportunités, comparativement aux Acadiens et aux Écossais. L'ethnomusicologue Dariush Zarbafian a donné son opinion sur l'importance de mener des travaux recherche en ethnomusicologie sur l'aspect structurel de la musique acadienne (ex. : rythme, mode, techniques de jeu, transmission), d'où l'importance d'effectuer au préalable un inventaire de l'ensemble des archives acadiennes et de mener des entretiens avec des musiciens et chercheurs.

Dans une séance plus décontractée, en soirée, qui avait pour titre Atelier et tour de chant: les traditions musicales acadiennes de Chéticamp, Kevin Johnson, étudiant au deuxième cycle à l'Université de l'Alberta, a présenté les résultats de son travail de recherche de premier cycle sur l'analyse mélodique (asymétrique) de quelques chansons du répertoire de Joseph à Athanase Larade de Chéticamp. Par la suite, Robert Deveaux de Chéticamp a interprété fidèlement un certain nombre de chansons du répertoire et dans le style (avec accompagnement au violon) de Joseph Larade. Le divertissement des conférenciers s'est poursuivi en veillée avec la musique folklorique acadienne du groupe Les Zorvenants du Cap-Breton, les chansons à répondre de Tess LeBlanc de l'Université Laval et du chansonnier Ken Chisholm de Sydney.

Lors de la sixième séance, qui avait pour titre Entrelacs acadiens et celtiques, Roy Johnstone de l' ̂̂le-du-Prince-Édouard (Î.-P.-É) a communiqué avec son violon les résultats de sa recherche informelle sur le répertoire des violoneux acadiens de son île ; le tout s'est conclu quand Roy Johnstone (violon), Karine Gallant (violon) et les membres du groupe Vishtèn ont interprété un pot-pourri de pièces jouées typiquement par les violoneux de l'Î.-P.-É. Autour d'une conférence chantée, Tess LeBlanc de l'Université Laval y est allée d'une comparaison des reels à bouche acadiens, québécois, irlandais et écossais. Evelyn Osborne de l'Université Memorial de Terre-Neuve s'est permis de faire un portrait du chevauchement entre les pièces radiophoniques écossaises du Cap-Breton et l'adoption de cette musique par les violoneux francophones de Terre-Neuve, au détriment de la tradition familiale française. 
Faisant partie de la septième séance, intitulée La danse au Cap-Breton et au Canada français, Pierre Chartrand du Centre Mnémo a dressé un tableau comparatif de « la danse acadienne dans le contexte des traditions du nord-est de l'Amérique du Nord ", ce qui n'est pas sans apporter de nombreuses pistes de questionnement intéressantes, notamment sur un possible apport des Amérindiens dans cette tradition. Sous forme de conférence dansée, Barbara LeBlanc de l'Université Sainte-Anne a présenté le résultat de ses recherches sur " la musique et les figures de quadrille à Chéticamp ». Mats Melin, de l'University of Limerick en Irlande, a présenté les conclusions de sa recherche, fruit de multiples enquêtes menées sur le terrain en NouvelleÉcosse. Son exposé traitait notamment de la façon de distinguer objectivement les styles des danseurs traditionnels acadiens, écossais et irlandais, ce que les pratiquants de la danse font de façon subjective.

Dans la huitième séance, un panel qui portait sur le violon traditionnel en Acadie, Devon Léger de Seattle a dressé un portrait de cette tradition (ex. : racine, rythme syncopé, toune croche, reel à bouche) et Sean O'Connell de Toronto y est allé avec un survol historique, ce qui a mené à une discussion sur ce qui caractérise la pratique des violoneux acadiens.

Pour la neuvième séance, L'Acadie dans son contexte nord-américain, le violoneux cadien David Greely, de l'Université de Louisiane, a illustré autant au violon qu'à l'oral les particularités de la tradition des violoneux cadiens et créoles. Gilles Losier de Montréal a exprimé avec son violon l'univers de la tradition musicale acadienne dans sa région natale de la Péninsule acadienne, au nord-est du Nouveau-Brunswick.

Présenté dans la dixième séance, titrée Aspects régionaux du violon traditionnel en Acadie, l'ethnomusicologue Meghan Forsyth de l'Université Memorial de Terre-Neuve a décrit les résultats préliminaires de ses enquêtes auprès des violoneux des Îles-de-la-Madeleine, rapportant, selon les récits de la jeune génération (moins de 50 ans), comment la transmission aurait été influencée autrefois par l'environnement des Madelinots (ex. : bruits du moteur d'un bateau de pêche, émissions radiophoniques). Au moyen de son violon et de documents audiovisuels, Colin Grant de Sydney a décrit généreusement les visages multiples de la pratique du violon traditionnel des Acadiens de la Baie Sainte-Marie et de Chéticamp. Avec des documents sonores d'époque en complément, Lisa Ornstein de Portland (Orégon) a tracé un portrait de l'héritage musical qu'a laissé Avila Leblanc des Îles-de-la-Madeleine, autant comme informateur que collecteur; elle a proposé la création d'un espace virtuel pour l'étude et la valorisation du patrimoine immatériel laissé par ce témoin.

Avec la onzième séance ayant comme titre Les traditions $d u$ CapBreton en évolution, Chris McDonald de CBu a défini la place du piano dans 
l'historiographie de la musique au Cap-Breton, et cela comme instrument d'accompagnement pour les violoneux de l'île. Liz Doherty, de l'Université d'Ulster en Irlande du Nord, a pour sa part présenté son projet de recherche sur les violoneux du Cap-Breton, qui devrait normalement aboutir à la publication d'un guide qui s'intitulerait The Cape Breton Fiddle Music Companion. Sa communication était entrelacée de parallèles entre la tradition au Cap-Breton et ailleurs dans le monde celtique, ce qui n'a pas laissé l'auditoire indifférent. En tentant d'y voir plus clair, Heather Sparling de CBU a présenté l'évolution et l'analyse de la pratique de la foulerie au Cap-Breton, en la comparant avec la tradition en Écosse et ailleurs au Canada. Elle a soulevé entre autres des questions sur la provenance de cette tradition ailleurs qu'en Écosse.

En avant-dernière séance, qui s'intitulait Les répertoires chantés, Tess LeBlanc de l'Université Laval a présenté son approche originale qui consiste à analyser la façon dont Allain Kelly, un chanteur traditionnel bilingue, combinait ses répertoires de langue anglaise et française. La communication de Margaret Steiner de l'Indiana University portait sur les prestations d'Allain Kelly au Miramichi Folksong Festival, afin d'analyser sa façon de s'identifier comme un bûcheron acadien de descendance irlandaise.

À la dernière séance, Regard vers l'avenir, Ian Russell de l'Université d'Aberdeen a proposé une synthèse, en faisant un parallèle entre le North Atlantic Fiddle Convention, de 2012 en Irlande du Nord, qui réunissait des musiciens irlandais catholiques et protestants, et le présent colloque, favorisant le dialogue entre anglophones et francophones sur la côte est du Canada.

En conclusion, deux projets ont été proposés, notamment un forum de discussion qui serait géré par Devon Léger, dans le but de faciliter les échanges d'idées et la création de projets communs ; et un groupe de participants, dirigé par Pierre Chartrand, qui se chargerait d'établir des archives virtuelles sur des musiciens légendaires comme Avila LeBlanc. Il y a eu également des discussions sur la possibilité d'établir un lien reliant les traditions francophones du Poitou, de l'Acadie et de la Louisiane que Marlène Belly coordonnerait. Les organisateurs souhaitent que l'édition du North Atlantic Fiddle Convention, qui se tiendra en 2015 au Cap-Breton, sache réanimer les débats sur le violon traditionnel en Acadie et dans les cultures celtiques. Enfin, plusieurs participants se sont donné rendez-vous à la réunion annuelle conjointe de la Société canadienne de musique traditionnelle et de l'Association canadienne d'ethnologie et de folklore à Sudbury en mai 2014. 